\title{
Gluteal-fold adipofascial perforator flap transposition for rectourethral fistula reconstruction
}

\author{
Keywords: Rectourethral fistula, rconstruction, gluteal-fold perforator flap, \\ adipofascial flap, perforator flap
}

Abstract: Objective: If a rectourinary fistula does not close spontaneously, it requires surgical closure. We present our experience of rectourethral fistula reconstruction using a gluteal-fold perforator flap, resulting in a successful outcome. Patient and Methods: The present was a 64-year-old man with prostate cancer who underwent radical prostatectomy. However, he developed rectourinary fistula, which required surgical closure. A dissection was undertaken to divide the fistula tract, and the rectal and urethral defect were closed. A $12.0 \times 3.0-\mathrm{cm}$ gluteal-fold adipofascial perforator flap was harvested and placed in the space between the rectum and urethra. Results: The viability of all flaps was favorable, without infection or necrosis. The patient could walk the next day, and was discharged two weeks later without fecaluria or liquid stool. Conclusions: We conclude that the glutealfold adipofascial perforator flap offers excellent functional advantages in rectourethral fistula reconstruction with minimal morbidity at the donor site. 


\section{Abstract}

2 Objective: If a rectourinary fistula does not close spontaneously, it requires surgical

3 closure. We present our experience of rectourethral fistula reconstruction using a

4 gluteal-fold perforator flap, resulting in a successful outcome. Patient and Methods:

5 The patient was a 64-year-old man with prostate cancer who underwent radical

6 prostatectomy. However, he developed rectourinary fistula, which required surgical

7 closure. A dissection was undertaken to divide the fistula tract, and the rectal and

8 urethral defect were closed. A 12.0×3.0-cm gluteal-fold adipofascial perforator flap

9 was harvested and placed in the space between the rectum and urethra. Results:

10 The viability of all flap was favorable, without infection or necrosis. The patient

11 could walk the next day, and was discharged two weeks later without fecaluria or

12 liquid stool. Conclusions: We conclude that the gluteal-fold adipofascial perforator

13 flap offers excellent functional advantages in rectourethral fistula reconstruction with

14 minimal morbidity at the donor site. 
16 Key words:

17 Rectourethral fistula reconstruction, gluteal-fold perforator flap, adipofascial flap 
19 Rectourinary fistula develops in 0.6 to $9 \%$ of patients after radical prostatectomy,

20 and seldom spontaneously heals. Generally, initial treatment required colostomy,

21 but this fails in more than $50 \%$ and these patients require surgical fistula closure [1] .

22 Various surgical procedures have been suggested for the repair of these fistulas [2].

23 A major technique to prevent recurrent rectourinary fistula has been with gracilis

24 muscle interposition [3]. However, it leads to high-level morbidity at the donor site,

25 including a long scar, wide resection area, and muscle loss.

26 We present a case of rectourethral fistula reconstructed with a gluteal-fold

27 perforator flap, resulting in a successful outcome.

\section{Patient and Methods}

29 A 64-year-old man was diagnosed with prostate cancer on biopsy and underwent

30 radical prostatectomy. On the 5th day after surgery, he develpoed fecaluria and

31 liquid stool. Retrograde cystourethrography showed the filling of the bladder and

32 the contrast in the rectum, which suggested the development of a rectourethral 
33 fistula (Figure 1). Although we created a colon stoma, the fistula had remained for

347 months. As liquid stool continued and did not decrease, surgical closure using a

35 gluteal-fold adipofascial perforator flap was planned.

36 The patient was placed in a supine position with the legs abducted. A horizontal

37 incision was made between the anus and scrotum. The dissection was undertaken

38 to divide the fistula tract, and all inflamed tissue was removed. The rectal and

39 urethral defect were closed (Figure 2). The location of the cutaneous perforator

40 vessels from the internal pudendal artery were identified on the medial side of the

41 ischial tuberosity preoperatively using a Doppler flowmeter. The adipofascial flap

42 was designed to include these points according to the size of the space between

43 the rectum and bladder.

44 Dissection of the flap was carried out lateral to medial in the fascial plane until the

45 perforator vessel could be seen. A $12.0 \times 3.0-\mathrm{cm}$ adipofascial flap including a

$467.0 \times 3.0-\mathrm{cm}$ hatchet-shaped skin flap was harvested (Figure 3). The adipofascial

47 flap was rotated and placed in the space between the rectum and urethra (Figures 4 , 
$48 \quad 5,6)$.

49 The viability of all flap was favorable, without infection or necrosis. Computed

50 tomography after 3 weeks showed that the adipofascial flap filled the soft tissue

51 defect between the rectum and bladder, and resolved the rectourinary fistula

52 (Figure 7). The patient could walk the next day, and was discharged two weeks

53 later without fecaluria or liquid stool. The urinary bladder catheter was removed 4

54 weeks after surgery. Two months later, the patient did not complain of

55 rectourethral fistula symptoms.

56 DISCUSSION

57 Although rectourinary fistula after radical prostatectomy is rare, if it does not

58 spontaneously close after colostomy, surgical closure should be considered.

59 Various surgical procedures have been suggested for the repair of these fistulas [2].

60 The rectal flap method with the York-Mason approach and gracilis muscle flap

61 interposition are the most common procedures. For radiated cases, gracilis muscle

62 interposition may be preferred, because bringing viable tissue to interpose between 
63 the rectum and urethra results in complete dissection between these two organs,

64 and both the rectal and urethral defects are repaired [1] . However, the gracilis

65 muscle is usually thin and the blood supply to the distal part of the muscle flap

66 becomes tenuous, which may lead to an insufficient flap volume to fill the cavity.

67 Furthermore, a gracilis muscle flap requires an extended incision for flap harvest

68 and mobilization [4].

69 The most significant advantage of the perforator flap is that there is no need to

70 sacrifice any main arteries; thus, there is minimal morbidity at the donor site [5].

71 Also, elevating the gracilis muscle flap sometimes causes prolonged sensory

72 disturbance of the medial thigh and gluteal regions, and the large muscle flap

73 involves a risk of developing hematoma [6]. On the other hand, the dissection of

74 adipofascial perforator flaps was carried out at a supra-fascial or fascial plane level,

75 bring free from nervous disturbance. Furthermore, the small incision reduced

76 intra-operative bleeding [6].

77 An ideal flap has a favorably vascularized skin paddle with the suitable thickness 
78 and width as the wound, which minimizes negative impacts on walking, creates a

79 natural esthetic appearance, and only requires a single-stage operation [7]. A

80 gluteal-fold adipofascial perforator flap fully satisfies these requirements.

81 Furthermore, surgeon can harvest the flap in the same operative field without

82 position changing, which is another advantage.

83 We conclude that the gluteal-fold adipofascial perforator flap offers excellent

84 functional advantages in rectourethral fistula reconstruction, with minimal morbidity

85 at the donor site.

86

87 Acknowledgments; none.

88 


\section{References}

90 1) Nyam DC, Pemberton JH. Management of iatrogenic rectourethral fistula. Dis

$91 \quad$ Colon Rectum 1999; 42: 994-997.

92 2) Boushey RP, McLeod RS, Cohen Z. Surgical management of acquired

93 rectourethral fistula, emphasizing the posterior approach. Can J Surg. 1998; 41:

$94 \quad 241-244$.

95 3) Ghoniem G, Elmissiry M, Weiss E, et al. Transperineal repair of complex

96 rectourethral fistula using gracilis muscle flap interposition-can urinary and

97 bowel functions be preserved? Journal of Urology. 2008;179(5):1882-1886.

98 4) Serafin D, Georgiade NG, Smith DH.Comparison of free flaps with pedicled flaps

99 for coverage of defects of the leg or foot.Plast Reconstr Surg. $1977 ; 59: 492-499$.

100 5) Ragoowansi R, Yii N, Niranjan N.Immediate vulvar and vaginal reconstruction

101 using the gluteal-fold flap: long-term results. Br J Plast Surg. 2004

102 Jul;57(5):406-410.

103 6) Lazzaro L, Guarneri GF, Rampino Cordaro E, et al.Vulvar reconstruction using a 
104 "V-Y" fascio-cutaneous gluteal flap: a valid reconstructive alternative in

105 post-oncological loss of substance.Arch Gynecol Obstet 2010;282(5):521-527.

106 7) El-Sabbagh AH.Skin perforator flaps: an algorithm for leg reconstruction.J

107 Reconstr Microsurg 2011;27:511-523.

110 Figure 1: Retrograde cystourethrography shows contrast fluid passing through

111 the rectum. (arrow (1): bladder, (2): rectum)

112 Figure 2: Intraoperative view showing dissection to divide the fistula tract and

113 the removal of all inflamed tissue.

114 Figure 3: Intraoperative view of the design of the gluteal-fold adipofascial

115 perforator flap (1): cutaneous perforator vessels from the internal pudendal

116 artery, (2): a $7.0 \times 3.0-\mathrm{cm}$ hatchet-shaped skin flap, (3) : a $12.0 \times 3.0-\mathrm{cm}$

117 adipofascial flap)

118 Figure 4: Intraoperative view of the elevated adipofascial perforator flap. 
119 Figure 5: View of the reconstructed rectourinary fistula using the gluteal-fold

120 perforator flap just after surgery.

121 Figure 6: Diagram of gluteal-fold adipofascial perforator flap transposition for the

122 rectourethral fistula technique.

123 Figure 7: Computed tomography after 3 weeks showing adipofasciaL flap

124 (arrow) transposition to separate the rectum and bladder, and resolution of the

125 rectourinary fistula. 


\section{Figure 1}

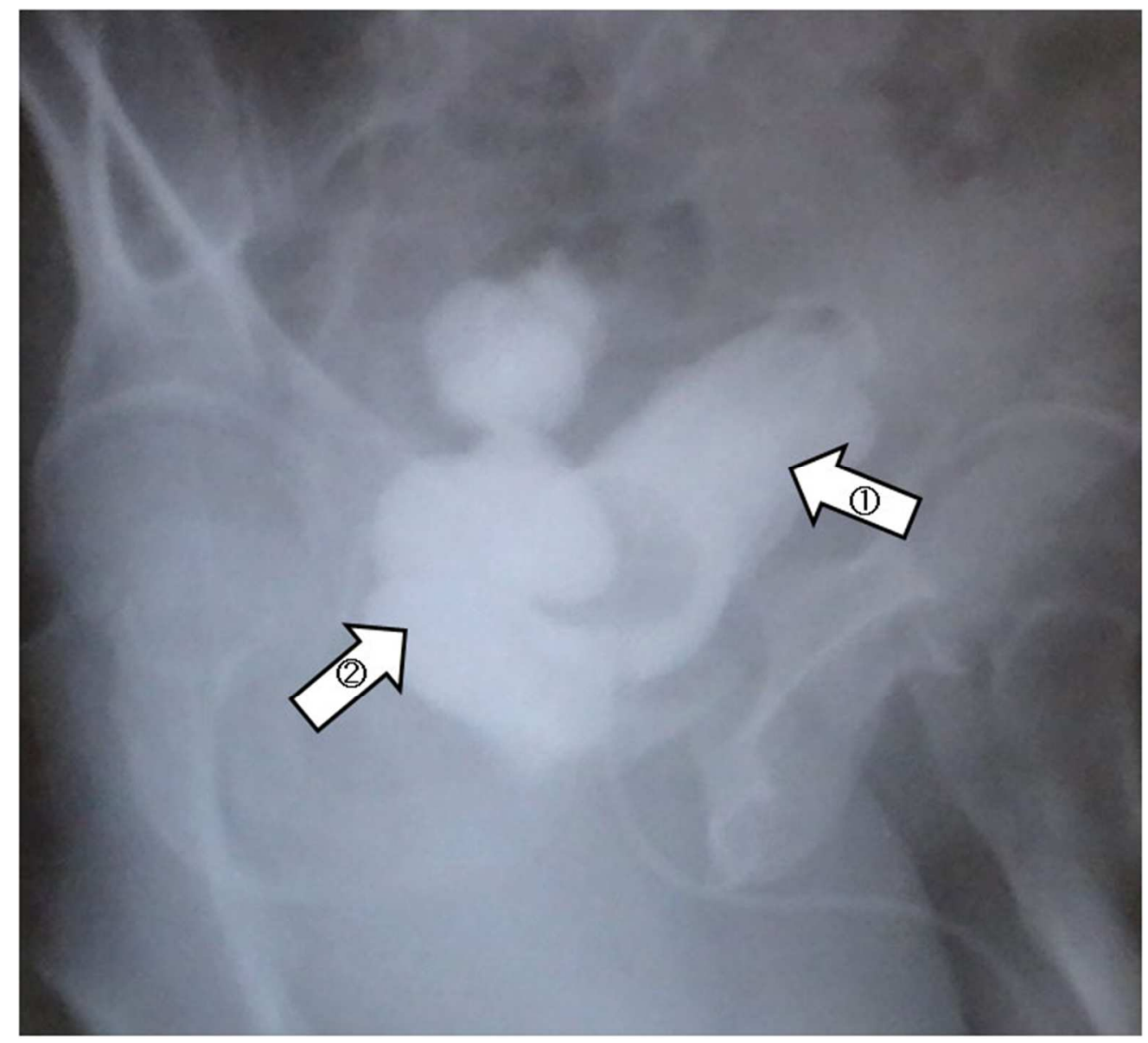

Retrograde cystourethrography shows contrast fluid passing through the rectum. (arrow (1): bladder, (2): rectum)

$60 \times 55 \mathrm{~mm}(300 \times 300 \mathrm{DPI})$ 


\section{Figure 2}

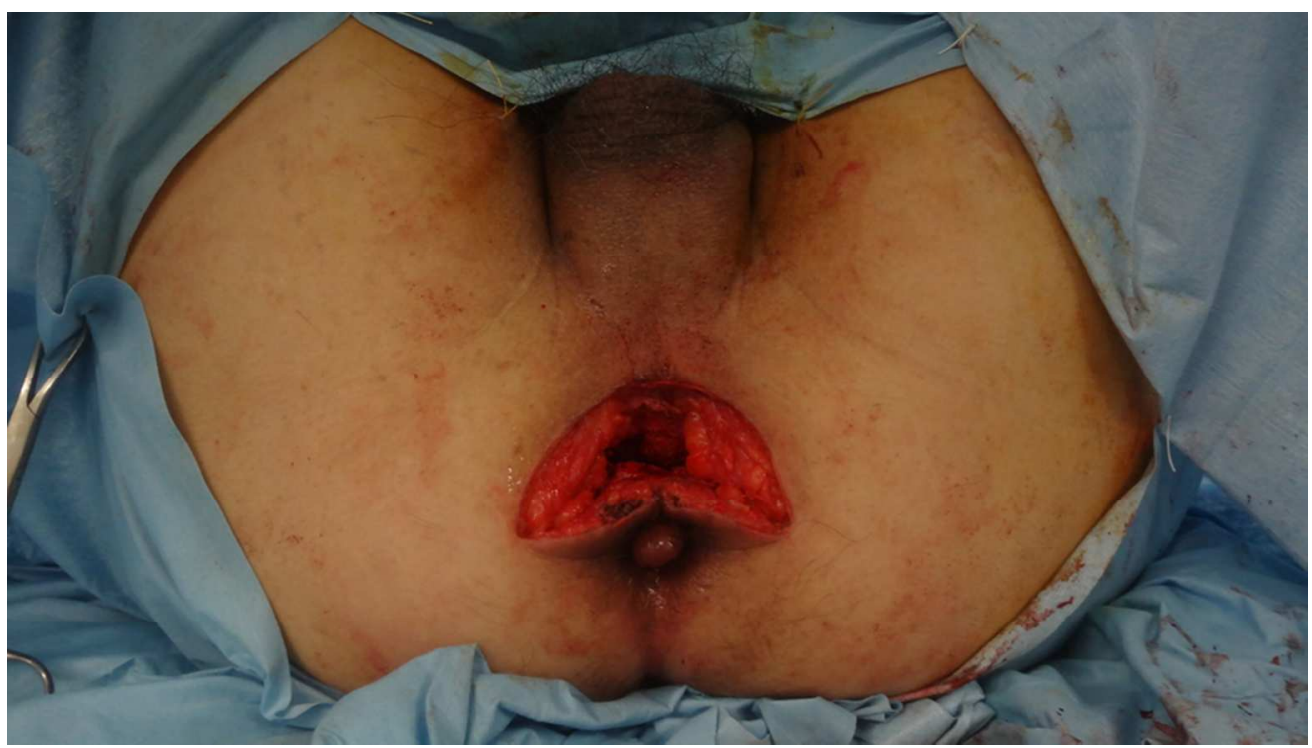

Intraoperative view showing dissection to divide the fistula tract and the removal of all inflamed tissue. $78 \times 44 \mathrm{~mm}(300 \times 300 \mathrm{DPI})$ 


\section{Figure 3}

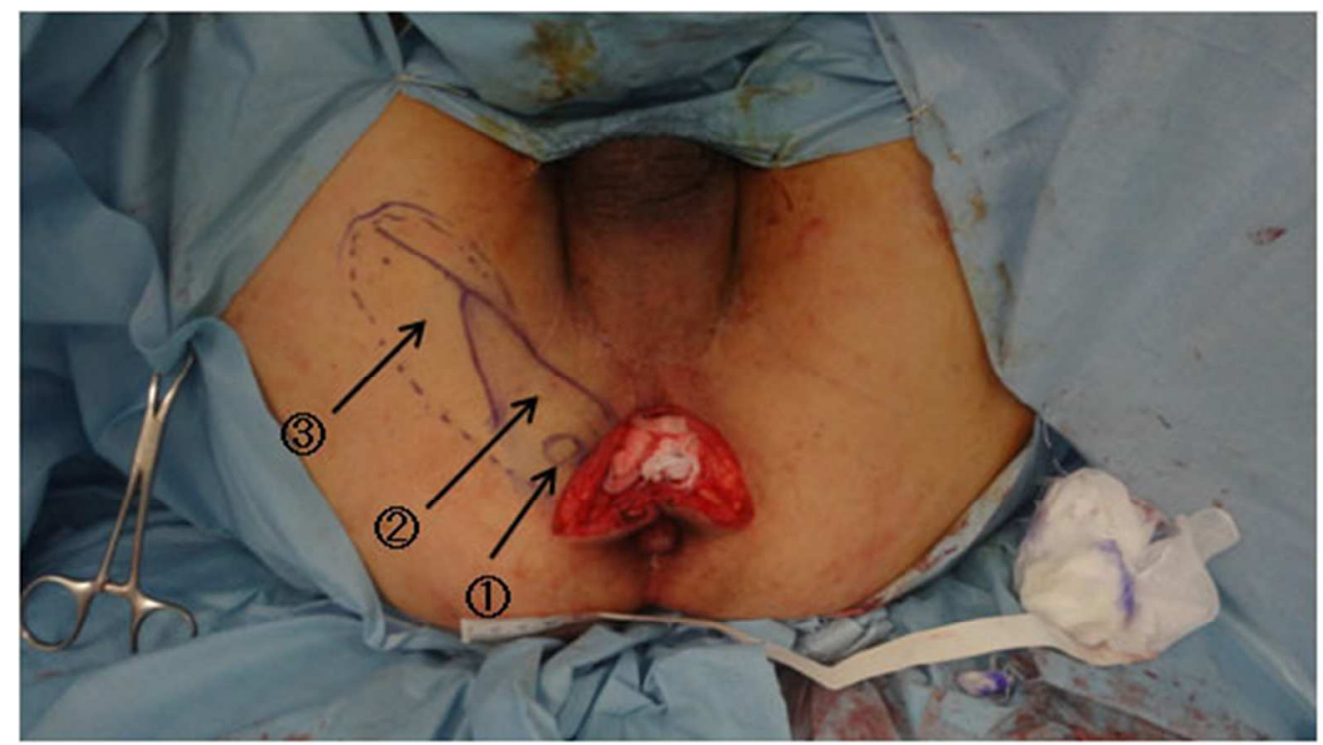

Intraoperative view of the design of the gluteal-fold adipofascial perforator flap (1): cutaneous perforator vessels from the internal pudendal artery, (2): a 7.0x3.0-cm hatchet-shaped skin flap, (3): a 12.0x3.0-cm adipofascial flap)

$44 \times 25 \mathrm{~mm}(300 \times 300$ DPI $)$ 
Figure 4

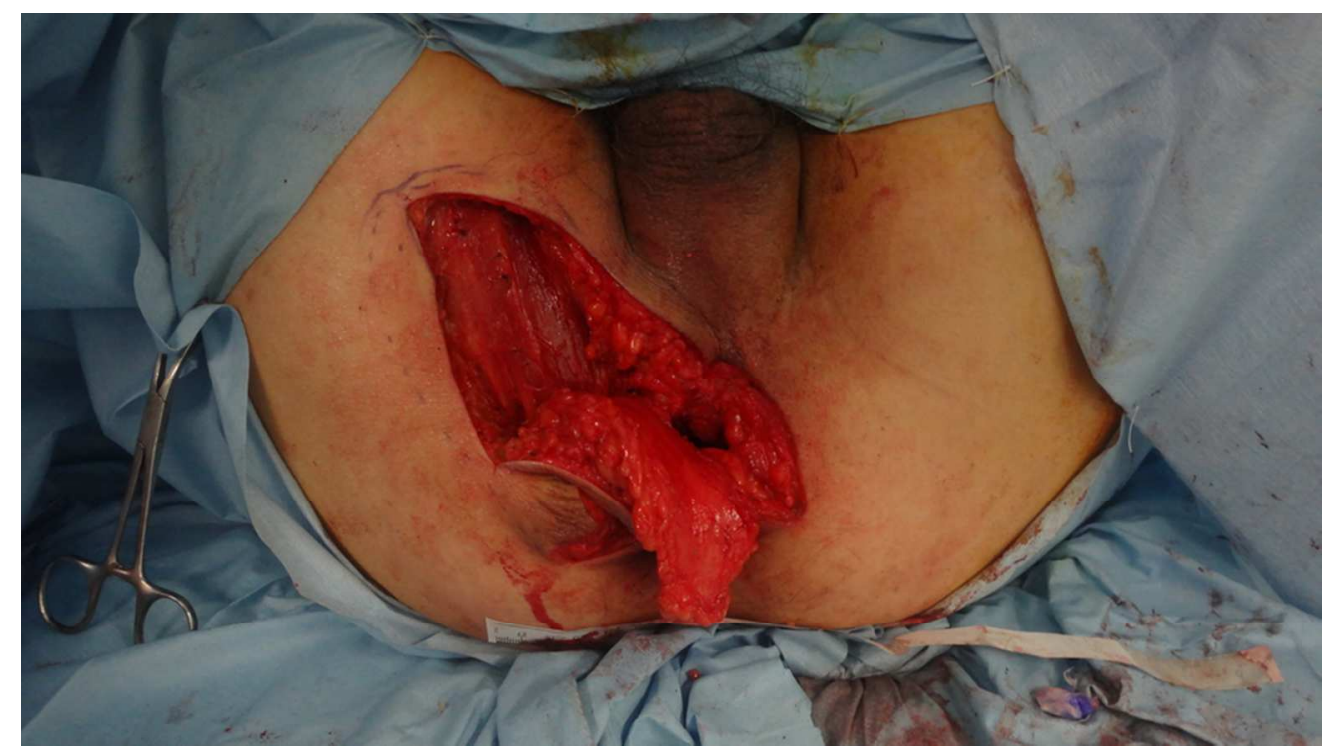

Intraoperative view of the elevated adipofascial perforator flap. $78 \times 44 \mathrm{~mm}(300 \times 300$ DPI $)$ 


\section{Figure 5}

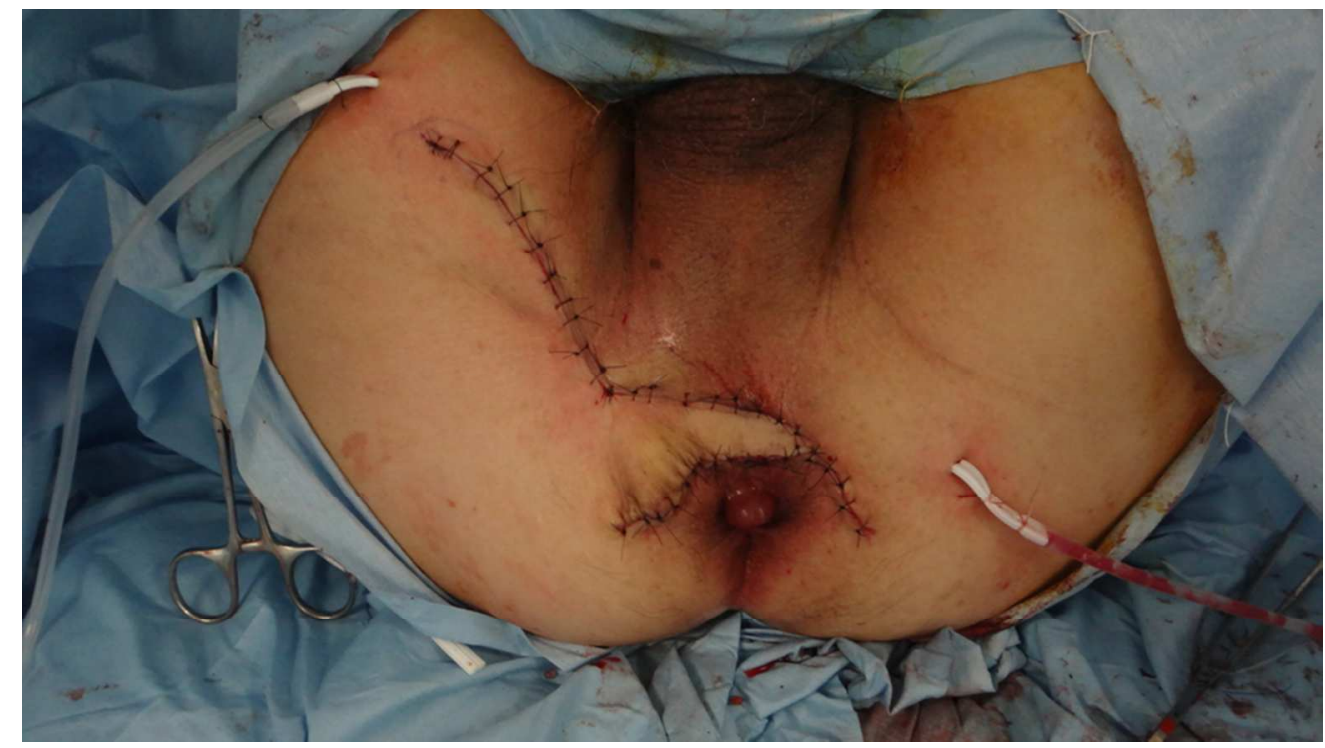

View of the reconstructed rectourinary fistula using the gluteal-fold perforator flap just after surgery. $78 \times 44 \mathrm{~mm}(300 \times 300 \mathrm{DPI})$ 
Figure 6

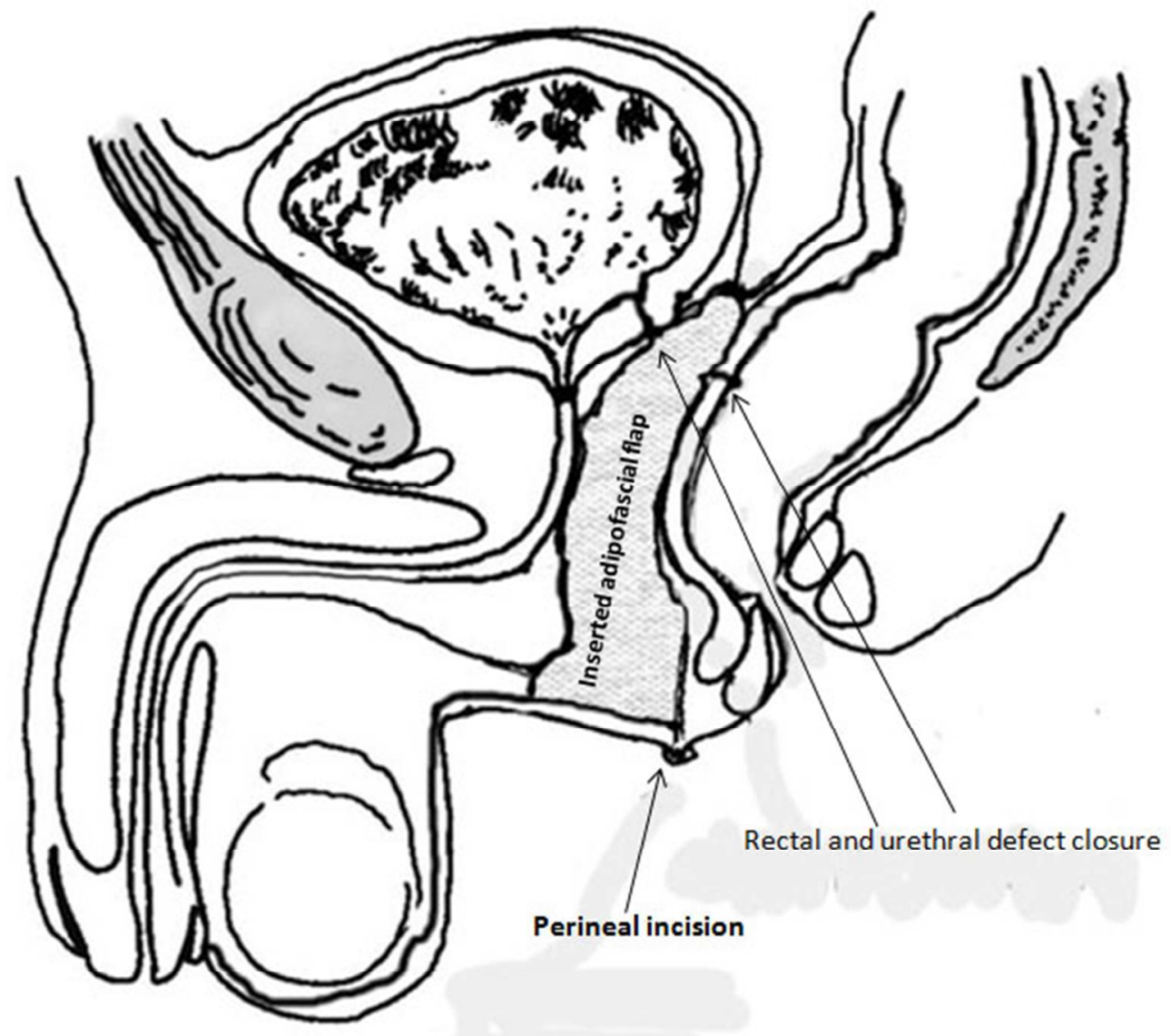

Diagram of gluteal-fold adipofascial perforator flap transposition for the rectourethral fistula technique. $217 \times 201 \mathrm{~mm}(72 \times 72 \mathrm{DPI})$ 


\section{Figure 7}

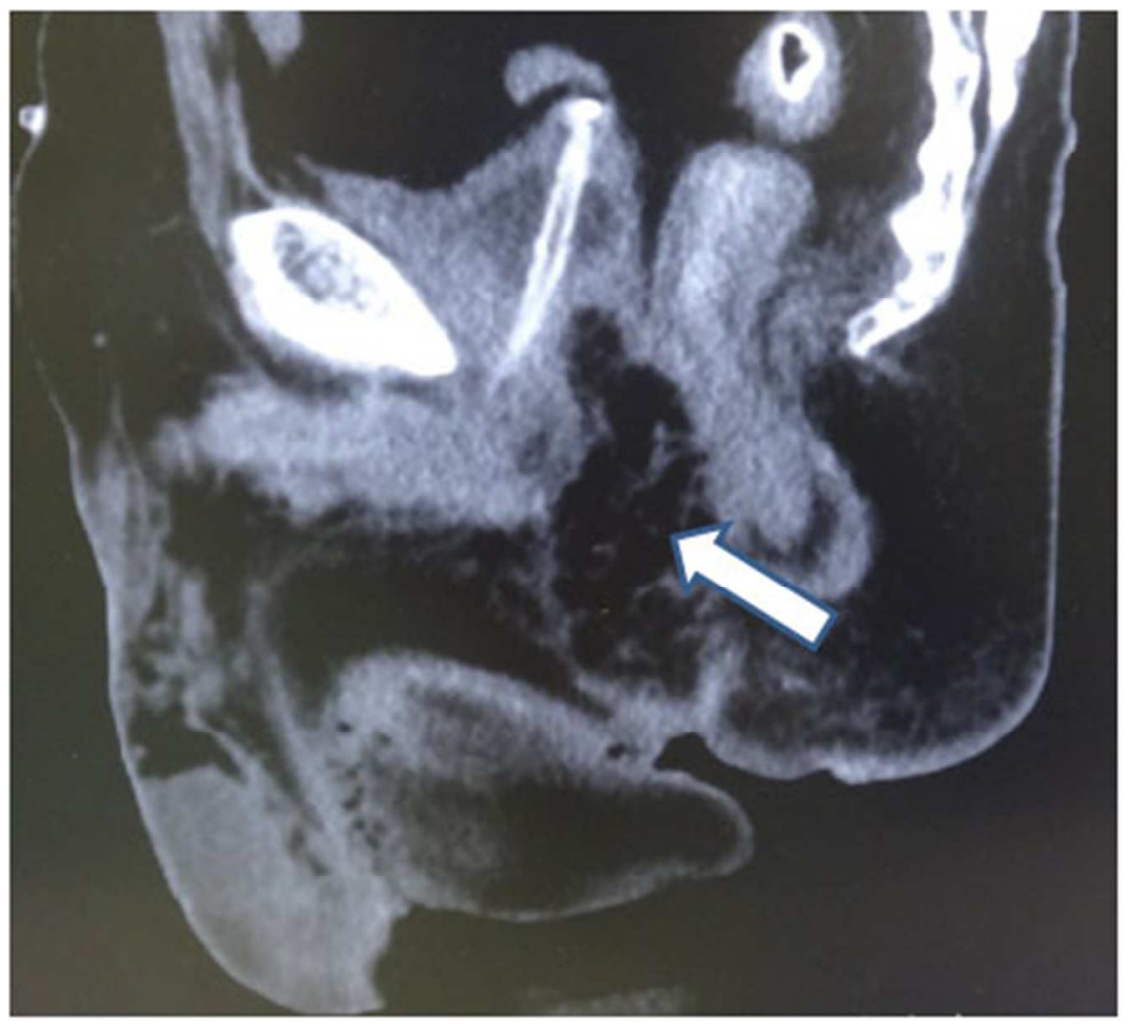

Computed tomography after 3 weeks showing adipofascial flap (arrow) transposition to separate the rectum and urethra, and resolution of the rectourinary fistula. $36 \times 32 \mathrm{~mm}(300 \times 300 \mathrm{DPI})$ 